\title{
OBSERVATIONS OF R CRB AND XX CAM
}

\author{
A. G. TOTOCHAVA
}

Abastumani Observatory, Abastumani, Georgia, U.S.S.R.

\begin{abstract}
The observations of R CrB and XX Cam obtained in 1971-1973 are described. Semiregular light variations with an amplitude of $0 m 15$ and a period of about 30 to 40 days were found during maximum brightness. In addition, fluctuations up to $0 m 2$ for about $2 \mathrm{~h}$ were observed.
\end{abstract}

Extended photoelectric and spectral research on the two stars $\mathrm{R} \mathrm{CrB}$ and XX Cam have been under way at the Abastumani Astrophysical Observatory since 1971.

The observations of $\mathrm{R} \mathrm{CrB}$ showed that at light maximum this star is characterized by semiregular light variations; the amplitude is equal to about 0.15 and the period is approximately 40 days (Totochava, 1973a). These values are consistent with Fernie's results (Fernie et al., 1972). From further observations of XX Cam the star was found to vary at its light maximum by approximately the same amplitude, but here the period is a little shorter (Totochava, 1973b). Neither color nor energy distribution changes in the continuum of the star accompany the light variations.

In 1972 the minimum of $\mathrm{R} \mathrm{CrB}$ was observed. At light minimum the star became bluer and it reddened strongly on the ascending branch. When the light of the star had decreased by about 4 mag., emission lines appeared in the spectrum. The distribution of the energy changed at the same time as the appearance of the emission lines.

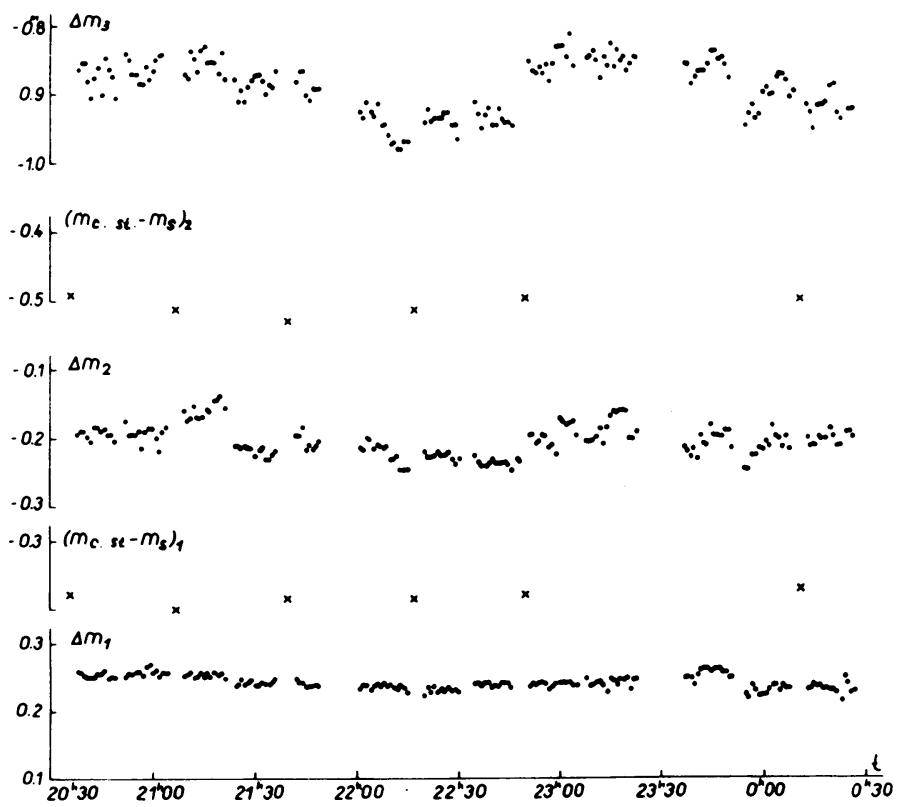

Fig. 1. The light curves of XX Cam obtained from simultaneous three-color observations on December 23-24, 1973 are shown. The light fluctuations are noticed only in blue and ultraviolet.

Crosses show the difference between the comparison star and the standard. 
With the view of revealing small light fluctuations during a single night, continuous observations of the light of XX Cam were begun in 1972. At first the observations were conducted with an ordinary single channel photometer, alternatively in each color $U, B, V$. From the light curves it is clear that there are some variations in yellow with an amplitude of about $0^{m} 1$ over several minutes. The fluctuations alternate with intervals when the star is very quiet. It is difficult to judge about light fluctuations in blue and ultraviolet, as we have far fewer observations in these regions than in yellow. In the autumn of 1973 the observations showed no light variations in yellow. At the same time continuous observations of this star with the help of a photoelectric three channel spectrocolorimeter were begun. The star was observed simultaneously in the following spectral ranges: $3350-3650 \AA, 4155-4280 \AA$ and $5120-5320 \AA$. From the light curves drawn on the basis of these observations it turned out that there are some synchronous fluctuations only in the ultraviolet and blue regions during certain nights (Figure 1). The amplitude of the fluctuations in ultraviolet is a little larger than in blue and it amounts to approximately $0^{m} 1$ during about two hours. On certain nights when

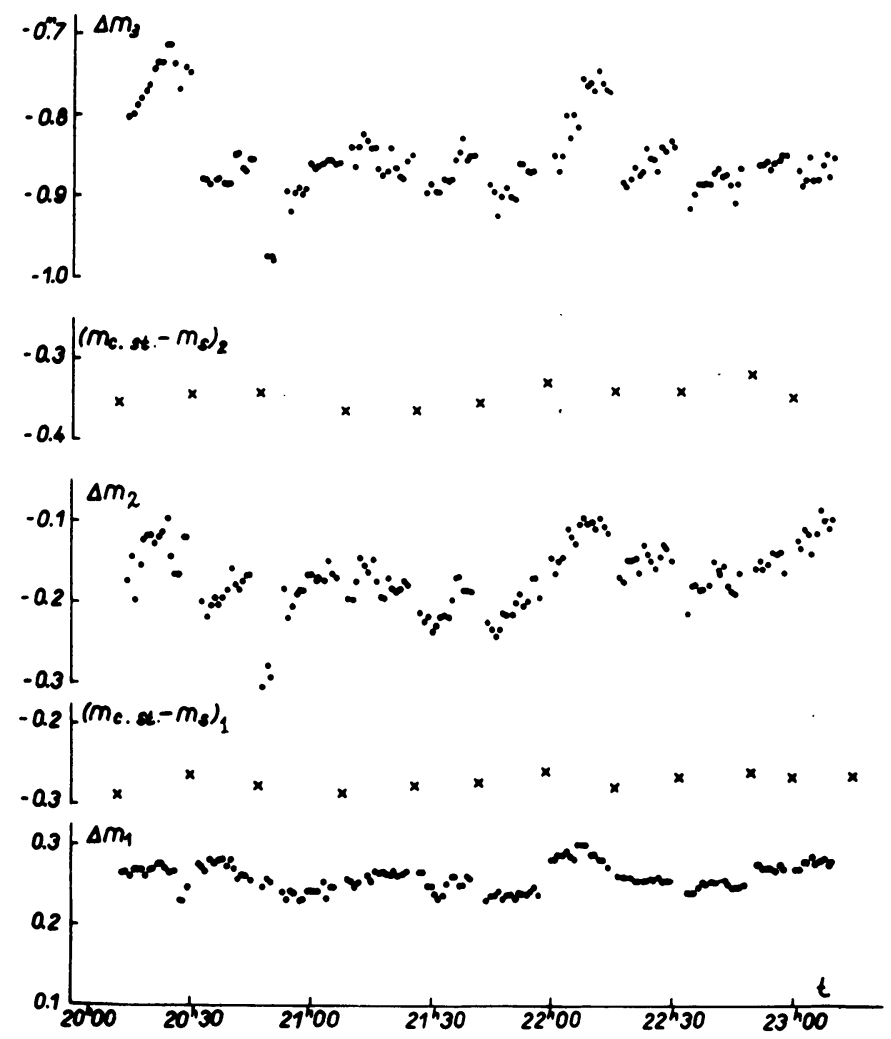

Fig. 2. The light curves of XX Cam obtained on 1974, February 15-16 are illustrated. The light fluctuations are observed in all three colors. 




Fig. 3. The figure shows the light curves of XX Cam obtained on February 25-26, 1974. There are no variations in any color.

the amplitude of fluctuations rises in these regions, the variation is noticeable in the yellow too (Figure 2). Sometimes there are no variations in any color (Figure 3).

At present, observations of $\mathrm{R} \mathrm{CrB}$ are being continued.

\section{References}

Fernie, J. D., Sherwood, V., and DuPuy, D. L.: 1972, Astrophys. J. 172, 383.

Totochava, A. G.: 1973a, Astron. Circ. U.R.S.S., No. 744.

Totochava, A. G.: 1973b, Astron. Circ. U.R.S.S., No. 791. 